\title{
LIFTING MODULAR REPRESENTATIONS OF FINITE GROUPS
}

\author{
BURTON FEIN ${ }^{1}$
}

Let $(\pi)$ be the maximal ideal of the ring $R$ of $P$-integral elements of an algebraic number field $K$, where $P$ is a prime of $K$ dividing the rational prime $p$. The natural homomorphism from $R$ to $\bar{K}=R /(\pi)$ induces a map $S \rightarrow \bar{S}$ from the set of representations by matrices with coefficients in $R$ of a finite group $G$ in to the set of representations of $G$ in $\bar{K}$. The lifting problem in modular representation theory is to determine whether for a given representation $T$ of $G$ in $\bar{K}$ there exists a representation $S$ of $G$ by $R$-matrices such that $\bar{S}=T$. In this paper we introduce a notion of lifting projective modular representations from characteristic $p$ to characteristic zero and show how this concept may be applied to the lifting problem.

Notation. Throughout this paper $G$ denotes a finite group of order $|G|$ and $K$ denotes an algebraic number field which is a splitting field for $G$. Let $p$ be a rational prime and let $R$ be the ring of $P$-integral elements of $K$, where $P$ is a prime of $K$ dividing $p$. Let $(\pi)$ be the maximal ideal of $R$ and set $\bar{K}=R /(\pi) . \bar{K}$ is a finite field of characteristic $p$ which is a splitting field for $G$. For $a \in R$, set $\bar{a}=a+(\pi) \in \bar{K}$. If $A=\left(a_{i j}\right)$ is a matrix with entries in $R$ ( $R$-matrix) we denote by $\bar{A}$ the matrix $\left(\bar{a}_{i j}\right)$. By a linear representation of $G$ in a field $L$ we shall understand a homomorphism from $G$ into $G L(m, L)$ for some $m$. By a projective integral representation (resp. projective modular representation) of $G$ in $R$ (resp. $\bar{K}$ ) we mean a map $T$ of $G$ into $G L(m, K)$ (resp. $G L(m, \bar{K})$ ) satisfying $T(1)=1_{m}, T(g) T(h)=\alpha(g, h)$ - $T(g h)$ where $\alpha(g, h) \in R$ (resp. $\bar{K})$ and $T(g)$ has entries in $R$ (resp. $\bar{K}$ ) for all $g, h \in G$. $\alpha$ is called the factor set associated with $T$. If $\alpha(g, h)$ $=\beta(g, h)=1$ for all $g, h \in G, T$ is a linear in tegral representation (resp. linear modular representation). We identify linear representations with projective representations having trivial factor sets. We refer the reader to [3] and [7] for the relevant theory.

Definition. Let $T$ be a projective modular representation of $G$ in $\bar{K}$ and let $\alpha$ be the associated factor set. $T$ is projectively liftable if there exists a projective integral representation $S$ of $G$ in $R$ with factor set $\beta$ such that $\bar{S}(g)=T(g)$ for all $g \in G$. If $\alpha(g, h)=\beta(g, h)=1$ for all $g, h \in G$ (i.e. $S$ and $T$ are linear representations), we say that $T$ is liftable.

Received by the editors November 1, 1966.

1 This research was supported by NSF Grant GP-5497. 
We emphasize that, according to the above definition, if we speak of a modular representation $T$ of $G$ in $\bar{K}$ being liftable to an integral representation $S$ of $G$ in $R$, then both $T$ and $S$ must be linear representations of $G$.

Lemma 1. Let $T$ and $W$ be projectively equivalent projective modular representations of $G$ of degree $m$ in $\bar{K}$. If $T$ is projectively liftable, then so is $W$.

Proof. By assumption there exists a function $\gamma$ from $G$ to $\bar{K}$ and a matrix $U \in G L(m, \bar{K})$ such that $U^{-1} T(g) U=W(g) \gamma(g)$ for all $g \in G$. Let $V \in G L(m, K)$ having entries in $R$ such that $\bar{V}=U$ and let $\alpha$ be a function from $G$ to $R$ such that $\bar{\alpha}^{-1}(g)=\gamma(g)$. det $V$ is a unit in $R$ so $V^{-1}$ has entries in $R$. Hence if $S$ is a projective integral representation which projectively lifts $T, V^{-1} S V \alpha$ is a projective lifting of $W$.

The next lemma will permit us to take finite extensions of $K$.

Lemma 2. Let $K_{1}$ be a finite extension of $K$ and let $R_{1}$ be the ring of $P_{1}$-integral elements of $K_{1}$, where $P_{1}$ is a prime of $K_{1}$ dividing the prime $P$ of $K$, i.e. $P_{1} \cap K=P$. Let $\left(\pi_{1}\right)$ be the maximal ideal of $R_{1}$ and view $\bar{K}$ as a subfield of $\bar{K}_{1}=R_{1} /\left(\pi_{1}\right)$. Let $T$ be an irreducible linear modular representation of $G$ in $\bar{K}$. If $T$ is liftable when viewed as a $\bar{K}_{1}$-representation, then $T$ is liftable as a $\bar{K}$-representation.

Proof. The lemma is a consequence of the fact that the decomposition matrix of $G$ for the prime $p$ does not depend on $K$ [3, Chapter 12].

THEOREM 1. Let $G$ be a finite group and suppose that $p \nmid\left|H^{2}\left(G, E^{*}\right)\right|$ where $E^{*}$ is the multiplicative group of an algebraic closure $E$ of $K$ and where $G$ acts trivially on $E^{*}$. Let $T$ be an irreducible linear modular representation of $G$ in $\bar{K}$ which is projectively liftable. Then $T$ is liftable.

ProOF. By assumption there is a projective integral representation $S$ of $G$ in $R$ with factor set $\alpha$ such that $\bar{S}(g)=T(g)$ for all $g \in G$ and $\bar{\alpha}(g, h)=1$ for all $g, h \in G$. Let $e$ be the order of $\alpha$ in $H^{2}\left(G, E^{*}\right)$. Then $\alpha$ is equivalent to $\alpha^{\prime}$ where $\alpha^{\prime}(g, h)$ is an eth root of unity for all $g, h \in G\left[3\right.$, p. 360]. There exists a function $\rho$ from $G$ to $E^{*}$ such that $\alpha^{\prime}(g, h)=\alpha(g, h) \rho(g h) \rho^{-1}(g) \rho^{-1}(h)$ for all $g, h \in G$. In view of Lemma 2 we may assume that $\rho(g) \in K$ for all $g \in G$. Let $\rho(g)=\pi^{\nu(g)} \gamma(g)$ where $\gamma(g)$ is a unit in $R$ and $\nu(g)$ is an integer. Since $\bar{\alpha}^{\prime}(g, h) \neq 0$, $\bar{\alpha}(g, h) \neq 0$ for all $g, h \in G, \nu(g)+\nu(h)=\nu(g h)$. Therefore $\alpha^{\prime}(g, h)$ $=\alpha(g, h) \gamma(g h) \gamma^{-1}(g) \gamma^{-1}(h)$ for all $g, h \in G$. Let $\gamma^{-1}(g)=\lambda(g)$ and set $Z(g)=\lambda(g) S(g)$ for all $g \in G$. $Z$ is a projective integral representation of $G$ in $R$ with factor set $\alpha^{\prime} . \bar{Z}$ is projectively equivalent over $\bar{K}$ to $T$. We may assume that $K$ contains a primitive $(|\bar{K}|-1)$-th root of 
unity $\delta$ over the rationals. Define a function $\mu$ from $G$ to the integers by $\bar{\lambda}(g)=\bar{\delta}^{\mu(g)}$ where $1 \leqq \mu(g) \leqq|K|-1$. Set $\eta(g)=\delta^{-\mu(g)}$ and let $V(g)$ $=\eta(g) Z(g) . V$ is a projective integral representation of $G$ in $R$ such that $\bar{V}=T$. The factor set $\beta$ associated with $V$ satisfies

$$
\beta(g, h)=\alpha^{\prime}(g, h) \eta^{-1}(g h) \eta(g) \eta(h)
$$

for all $g, h \in G$. Since $\beta(g, h)$ is a root of unity with $\bar{\beta}(g, h)=1$ for all $g, h \in G$, we see that $\{\beta\}$ has order $p^{b}$ in $H^{2}\left(G, E^{*}\right)$. Since $p \nmid\left|H^{2}\left(G, E^{*}\right)\right|$ by assumption, $\beta$ is equivalent to the unit factor set. Therefore there is a function $\tau$ from $G$ to $E^{*}$ such that

$$
\beta(g, h)=\tau(g h) \tau^{-1}(g) \tau^{-1}(h)
$$

for all $g, h \in G$. As before we may assume that $\tau(g) \in R$ for all $g \in G$. Let $W(g)=\tau(g) V(g)$. $W$ is a projective integral representation of $G$ in $R$ and $\bar{W}(g)=\bar{\tau}(g) \bar{V}(g)=\bar{\tau}(g) T(g)$ for all $g \in G$. Since $\bar{\beta}(g, h)=1$ for all $g, h \in G, \bar{W}$ is a linear modular representation of $G$ in $\bar{K}$. Let $\bar{\tau}(g)=\bar{\delta}^{\theta(g)}$ where $1 \leqq \theta(g) \leqq|K|-1$. Let $M(g)=\delta^{\theta(g)} W(g)$ for all $g \in G$. $M$ is a linear integral representation of $G$ in $R$ and $\bar{M}=T$ and so $T$ is liftable.

We refer the reader to [3, p. 361] for the definition and construction of a representation-group of a finite group with respect to an algebraically closed field (see also [7]). A representation-group $G^{*}$ of $G$ with respect to $E$ is a central extension of $G$ with kernel $A \cong H^{2}\left(G, E^{*}\right)$ with the following property: Let $T$ be a projective representation of degree $m$ of $G$ in $E$. Then if $\left\{u_{g}: g \in G\right\}$ is a set of coset representatives of $A$ in $G^{*}$, there exists a projective representation $T^{\prime}$ of $G$ in $E$ which is projectively equivalent to $T$ and a linear representation $S$ of $G^{*}$ with $S(a) \in E^{*} \cdot 1_{m}$ for all $a \in A$ and $S\left(u_{g}\right)=T^{\prime}(g)$ for all $g \in G$. If $S$ and $T^{\prime}$ have this relationship we say that $S$ linearizes $T^{\prime}$. Let $\bar{E}$ be an algebraic closure of $\bar{K}$. If $p \nmid\left|H^{2}\left(G, E^{*}\right)\right|$, then a representationgroup for $G$ with respect to $E$ is also one with respect to $\bar{E}$ [1, Satz 2].

Definition. We say that $G$ has property $(p, m)$ if every irreducible linear modular representation of degree $m$ of $G$ in $\bar{K}$ is liftable.

In view of Lemma 2 we see that property $(p, m)$ does not depend on the splitting field chosen.

Lemma 3. Let $G^{*}$ be a representation-group for $G$ with respect to $E$ and suppose that $K$ is a splitting field for both $G$ and $G^{*}$. Assume also that $p \nmid\left|H^{2}\left(G, E^{*}\right)\right|$ and $G^{*}$ has property $(p, m)$ (with respect to $\bar{K}$ ). Let $T$ be an absolutely irreducible projective modular representation of degree $m$ of $G$ in $\bar{K}$. Then there exists a finite extension $K_{1}$ of $K$ such that, in the context of Lemma 2, $T$ is projectively liftable (to an $R_{1}$-representation) when viewed as a representation of $G$ in $\bar{K}_{1}$. 
Proof. As noted above $G^{*}$ is a representation-group for $G$ with respect to both $E$ and $\bar{E}$. Tis projectively equivalent over $\bar{E}$ to an irreducible projective representation $T^{\prime}$ of $G$ in $\bar{E}$, where $T^{\prime}$ is linearizable. Let $S$ be a linear representation of $G^{*}$ in $\bar{E}$ which linearizes $T^{\prime}$. Hence there is a finite extension $L$ of $\bar{K}$ such that the entries of $S(h)$ and $T^{\prime}(g)$ lie in $L$ for all $h \in G^{*}$ and all $g \in G$ and $T^{\prime}$ is projectively equivalent to $T$ over $L$. In view of Lemma 1 we may assume that $T=T^{\prime}$. There exists a finite extension $K_{1}$ of $K$ containing a valuation ring $R_{1}$ with maximal ideal $\left(\pi_{1}\right)$ such that $L=\bar{K}_{1}=R_{1} /\left(\pi_{1}\right)$. Since $K_{1}$ is a splitting field for $G^{*}, G^{*}$ has property $(p, m)$ with respect to $\bar{K}_{1} . S$ is an irreducible linear modular representation of degree $m$ of $G$ in $\bar{K}_{1}$ and so $S$ is liftable. Let $V$ be a linear integral representation of $G^{*}$ in $R_{1}$ with $\bar{V}(h)=S(h)$ for all $h \in G^{*}$. $G^{*}$ is a central extension of $G$ with kernel $A$, say. If $a \in A$, then $S(a)=\lambda(a) 1_{m}$ with $\lambda(a) \in \bar{K}_{1} . \quad V(a)$ $=\mu(a) 1_{m}$, with $\bar{\mu}(a)=\lambda(a)$. Let $\left\{u_{g}: g \in G\right\}$ be a set of coset representatives of $A$ in $G^{*}$. Set $U(g)=V\left(u_{g}\right)$ for all $g \in G$. Then $U$ is a projective lifting of $T, T$ being viewed as a $\bar{K}_{1}$-representation. This proves the result.

THEOREM 2. Let $G$ be a finite group possessing an abelian normal subgroup $A$ such that every proper subgroup of $B=G / A$ is p-solvable. Suppose also that $p \nmid\left|H^{2}\left(G, E^{*}\right)\right|, p \nmid\left|H^{2}\left(B, E^{*}\right)\right|$ and that the algebraic number field $K$ is now a splitting field for all subquotients of $G^{*}$ and $B^{*}$, where $G^{*}$ and $B^{*}$ are some two representation-groups of $G$ and $B$ respectively. If $B^{*}$ possesses property $(p, m)$ (with respect to $\bar{K}$ ) then so does $G$.

Proof. Let $T$ be an irreducible linear modular representation of degree $m$ of $G$ in $\bar{K}$ and let $T_{\mathrm{A}}$ denote the restriction of $T$ to $A$. By Clifford's Theorem we have

$$
T_{A} \sim e\left(T_{1}+\cdots+T_{r}\right)
$$

where $T_{1}, \cdots, T_{r}$ are inequivalent conjugate irreducible linear representations of $A$ in $\bar{K}$. Since $\bar{K}$ is also a splitting field for $A$ and $A$ is abelian, each of the $T_{i}$ 's has degree one.

Case 1. $r>1$. Let $I$ be the inertia group of $T_{1}$ in $G$ and view $e T_{1}$ as a representation $W$ of $I$. $I \supset A$ and $I$ is a proper subgroup of $G$ so $I$ is $p$-solvable. $W$ is an irreducible linear modular representation of $I$ in $\bar{K}$ such that $W^{G} \sim T$, where $W^{G}$ is the induced representation of $W$ to $G[3$, p. 348]. Since $I$ is $p$-solvable, there exists an irreducible linear integral representation $S$ of $I$ in $R$ with $\bar{S}(g)=W(g)$ for all $g \in I$ [6, Theorem 6]. Let $V=S^{G} . \bar{V}$ and $\bar{S}^{G}=W^{G} \sim T$ have the same character and the same degree. Since $T$ is irreducible, $\bar{V} \sim T$ and so $T$ is liftable. 
Case 2. $r=1$. We have $T \sim C \times D$ (Kronecker product) where $C, D$ are irreducible projective representations of $G$ in $\bar{E}$ such that $C$ is one-dimensional and $D(g)=1_{m}$ for $g \in A$ [3, Theorem 51.7]. Extending $K$ if necessary, we may assume that the entries of $C(g), D(g)$ lie in $\bar{K}$ for all $g \in G$, that $T$ is equivalent to $C \times D$ over $\bar{K}$, and that $D$ is projectively liftable with respect to $\bar{K}$. We refer to Lemmas 2 and 3 to justify this step. $C$ is projectively liftable since $C$ is a 1-dimensional representation. Hence $C \times D$ is projectively liftable and it follows from Theorem 1 that $T$ is liftable.

REMARK. The hypothesis that $p \nmid\left|H^{2}\left(G, E^{*}\right)\right|$ is satisfied, for example, when $G$ has a cyclic Sylow $p$-subgroup [5, p. 49]. If $p$ is a prime, $p \geqq 5$, then $L F(2, p)$ is a minimal simple group [4, Chapter 12]. The hypotheses of Theorem 2 are satisfied when $p \nmid|A|, B=G / A$ $\cong L F(2, p)$ where $p \geqq 5, \bar{K}$ has characteristic $p$, and $m=(p-1) / 2$ or $(p+1) / 2[2$, p. 590$]$.

\section{REFERENCES}

1. K. Asano, M. Osima and M. Takahasi, Über die Darstellung von Gruppen durch Kollineationen im Körper der Charakteristik p, Proc. Phys. Math. Soc. Japan 19 (1937), 199-209.

2. R. Brauer and C. Nesbitt, On the modular characters of groups, Ann. of Math. 42 (1941), 556-590.

3. C. W. Curtis and I. Reiner, Representation theory of finite groups and associative algebras, Interscience, New York, 1962.

4. L. Dickson, Linear groups, Leipzig, 1901.

5. I. Schur, Über die Darstellungen der endlichen Gruppen durch gebrochene lineare Substitutionen, J. für Math. 127 (1904), 20-50.

6. R. Swan, The Grothendieck ring of a finite group, Topology 2 (1963), 85-110.

7. K. Yamazaki, On projective representations and ring extensions of finite groups. J. Fac. Sci. Univ. Tokyo Sect. I 10 (1964), 147-195.

University of California, Los Angeles 